\title{
ARCHITECTURAL BUILDING AERODYNAMICS OF TALL STRUCTURES WITH THE BLEEDING EFFECT AND WIND ENERGY SELECTION
}

\author{
Nick Vatin $^{1}$, Sergey Isaev ${ }^{2}$, Sergey Guvernyik ${ }^{3}$, Vladimir Gagarin ${ }^{4}$, Boris Basok $^{5}$, Yulia Zhukova ${ }^{6}$ \\ ${ }^{1,2}$ Department of Construction of Unique Buildings and Structures, Engineering Construction Institute, Saint- \\ Petersburg State Polytechnical University, Polytechnical Str. 29, 195251 Saint-Petersburg, Russia \\ ${ }^{3}$ Institute of Mechanics, Lomonosov Moscow State University, Michurin Av. 1, 119192 Moscow, Russia \\ ${ }^{4}$ National Research University, Moscow State University of Civil Engineering, Yaroslavskoe Shosse, 26, \\ 129337Moscow, Russia \\ ${ }^{5}$ Institute of Technical Thermophysics of the National Academy of Science of Ukraine, Zhelyabov Str. 2a, Kiev, Ukraine \\ ${ }^{6}$ A. V. Luikov Heat and Mass Transfer Institute of National Academy of Sciences of Belarus, P. Brovka Str. 15, 220072 \\ Minsk, Republic of Belarus
E-mail.:1vatin@mail.ru; ${ }^{2}$ isaev3612@yandex.ru (correspondingauthor); ${ }^{3}$ guv@mail.ru; ${ }^{4}$ gagarin@mail.ru; ${ }^{5}$ basok@ittf.kiev.ua; ${ }^{6}$ julia_zhukova@rambler.ru

\begin{abstract}
The material presented here demonstrates the experience gained over the past two decades. The emphasis is made on solving the fundamental problems of vortex aerohydromechanics and thermal physics that are related to the construction science. One of the major objectives of this work is to arrange the research directions according to this subject with the intent of showing their inter-discipline character.
\end{abstract}

Keywords: High-rise building, wind influence, unsteady flow, acting forces, pulsation, numerical simulation, verification, multiblock grids, models of turbulence, URANS, codes

\section{Introduction}

Over the recent years comprehensive computational and experimental research of architecture and building aerothermodynamics and acoustics of high-rise and largespan constructions and urban development (Isaev et al. 2013 (a); Isaev et al. 2013 (b)) has been expanded in a number of teaching and learning centers of the Russian Federation, the Ukraine, and Belarus. In particular, the meteorological-type wind tunnel involving the simulation of atmosphere boundary layer has been designed and is being built at the Moscow State Building University (MSBU). A number of projects on the development, testing, and use of high-performance computing systems with parallel architecture based on multiblock computational technologies (MCT) and mesoscale models of wind effects on high-rise building constructions have been initiated and are being implemented. The actual research direction in environmental aerodynamics (Lawson 2001) associated with architecture and building aerothermodynamics has been formulated and is now under development. Some of its fundamental items are illustrated in the paper.

The problem of numerical simulation of turbulent transfer is one of the fundamental tasks of computational aerodynamics in civil engineering. One of the modern engineering approaches to the solution of this task is associated with the use of semi-empirical turbulence models for closing Reynolds averaged Navier-Stokes equations. Among the most correct and promising methods are Large Eddy Simulation (LES) and Detached Eddy Simulation (DES), although the latter requires large computational resources. The models as given above are incorporated into the catalogue of universal commercial codes (e.g., ANSYS Fluent, Star CD). So, they have become widely used. However, the specificity of the problems for prediction of the wind loading on high-rise buildings calls for a thorough discretization of computational domains in the boundary and shear layers, vortex street zones, and in jet-vortex flow. But this is not always taken into account in computations relying on the universal codes. Sometimes the study of the transient process, for example, the wake and vortex streets interference behind an ensemble of bodies is replaced with the solution of the quasi-steady problem governed by the Reynolds steady-state equations.

The mathematical model as given in the present study is a system of the Reynolds averaged Navier-Stokes equations for viscous incompressible liquid along with the transport equations for turbulence characteristics. For the equations to be closed, Menter's shear stress transfer model (MSST) (Menter 1993) is adopted. The above MSST model is preferable for computations of vortex and separated flows (Menter et al. 2003).

\section{The research directions}

The concept of separated flow control as applied to the problems of external and internal aerohydrodynamics: drag reduction

The first group of research works is a series of investigations, which began in 1973 and was concerned with aerodynamics of bluff bodies when implementing the concept of separated flow control in developing circulation zones (Belov et al. 1989). The example of the tandem of bodies - protruding co-axial disc-provided cylinder is taken from automotive practice - cab-trailer (Sander's patent 1966). Its profile drag falls practically to zero due to the trapping of an extremely powerful toroidal vortex in the gap between the disc and the cylinder end (the backflow velocity appears to be of the order of the incoming flow velocity). The vortex at the angle of attack is not simply deformed, but there appears a complex jet-vortex structure in the spatial separated flow zone - 
column-shaped swirled flows on the leeward side of the cylinder end. The analysis of the pictures of liquid spreading over the end and the computer visualizations of the spatial vortex structure explains the stabilization effect (Baranov et al 2003). Among the dependences of integral characteristics of the disc-provided cylinder is first of all the availability of the negative force acting upon the cylinder and compensating the load upon it, as well as the onset of the restoring moment both for the end and the disc (by the order of magnitude less). It should be noted that the projects on separated flow control have been executed in collaboration with some of the organizations of the Russian Federation, the Ukraine, and Belarus over the past decades. They were related not only to the mentioned models of bodies but also to aircrafts and industrial objects. So, in the early 90's the work was implemented for the B.E. Vedeneev All-Russian Scientific-Research Institute of Hydraulic Engineering to substantiate the technology of reducing droplet spraying under the wind action upon cooling towers using the fences that decrease the flow nonuniformity in the vicinity of lower windows (Bungov et al 1998). The concept of minimizing the drag is used in architecture and building aerodynamics when designing a block of buildings.

\section{Effective surface reliefs - vortex generators, power engineering}

The next series of fundamental works is devoted to G. A Dreitser's terminology (Kalinin et al 1998) on effective heat transfer surfaces. Indeed, these are nonsmooth surfaces with organized roughness - reliefs with dimples and protrusions. In a spherical dimple, complex separated flow occurs. As the depth of the dimple is increased, the two-cell vortex structure is replaced by the single-cell vortex, one with substantial heat transfer enhancement (Isaev et al. 2010 (a)). New reliefs with oval dimples, which generate single-cell vortices (Bystrov et al. 2005), are proposed. The recent studies of extended oval dimples have revealed their high efficiency both due to forming spiral-like spatial vortices and due to decreasing separated flow zones characteristic for low heat transfer (Isaev et al. 2010 (b)). It has been established that oval dimples surpass the spherical ones almost by a factor of three Reliefs with staggered oval dimples are substantiated. It is evident that dimples and protrusions can be considered as model elements of largescale reliefs directly relating to construction sites.

\section{Mesoscale models - computation of the wind near airports}

The next series of investigations is related to aviation meteorology and is associated with predicting the hazardous wind shift in the vicinity of airports. It is known that at the takeoff - landing, aircrafts are flying on the glide path (Babaskin et al. 2002). The emphasis has been made both on developing mesoscale models of shearing flows of the wind that develop under the action of orographical roughness and on filling the databases of the wind speed on the glide path. The airports in Pulkovo and in the city of Nalchik have been considered. The approach for modeling the formation of the surface wind to be simulated in the wind tunnel is developed. As the example of implementing the mesoscale model (Babaskin et al. 2008), the influence of the urban development in the vicinity of the airport in the city of Nalchik on the formation of the wind shear with regard to the relief (on the map) is assessed.

\section{Wind engineering, vortex technologies}

Vortex cells are formed due to suction in cavities with the energy action upon circulating flows (Ermishin, Isaev 2003). As a result, it appears possible to change the character of flow around large-thickness bodies: its separated behavior is replaced by practically nonseparated one. Drag reduction and lift increase are the result of such action. Wind engineering is illustrated by the construction of low-noise wind engines designed at TsAGI (Isaev et al. 2003).

\section{Tunnel ventilation}

The problem of smoke formation in Metro halls at fire aboard the train by the request of VNII PO was considered in 1999. The series of investigations into the ventilation of road tunnels (for example, Lefortovo tunnel), cockpits (Usachov et al. 2004; Baranov et al. 2005), which have been built over the past decade, is directly related to the solution of the problems associated with the ventilation and conditioning of building rooms.

\section{Wind loads at constructions. Load reduction due to the throttling effect. Drag reduction when extracting wind energy}

The problem of reducing alternating wind loads at high-rise buildings was stated in 2000 at the colloquium on aerodynamics of bluff bodies in Bochum (Isaev et al. 2002). The problem was solved by using the throttling effect associated with air cross-over from high-pressure regions to low-pressure ones in the flow around the cylinder.

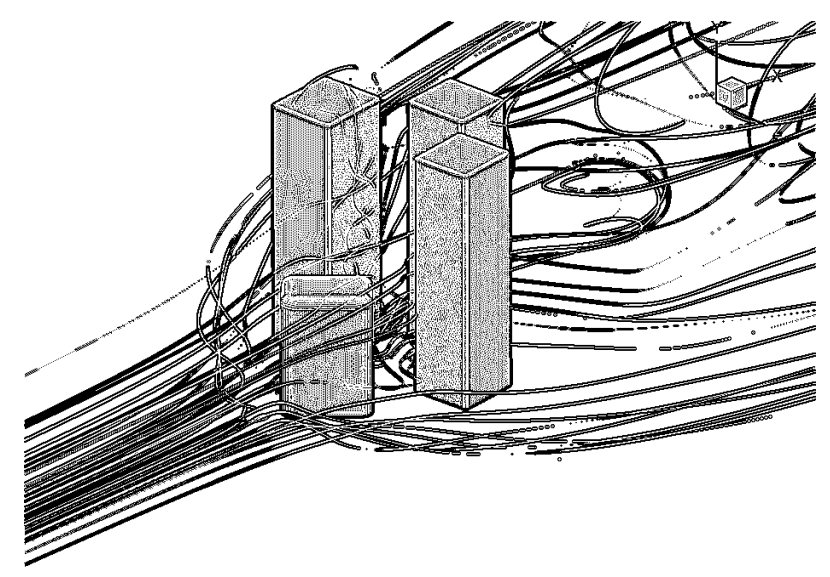

Fig. 1. Flow around the model of the micro-district "EKO". 
In 2006, the solution to the test problem of spatial air flow around a parallelepiped on the plane was presented at the meeting of Moscow City architecture council, and in 2007, a real development project of Moscow (a block of high-rise buildings "EKO") (Isaev et al. 2003) was computed. In the current research, averaged and pulsation characteristics in all-round flow around the block of buildings are analyzed. The ventilation of the space between buildings is investigated by visualizing the changes in averaged flow velocity fields at various heights from the ground. The vortex picture of flow around high-rise buildings is similar to that of a model construction in the form of cube, and a saddle-shaped vortex develops in front of buildings. The system of obstacles is blown by winds and powerful vortex structures (Fig. 1) are formed inside the micro-district.

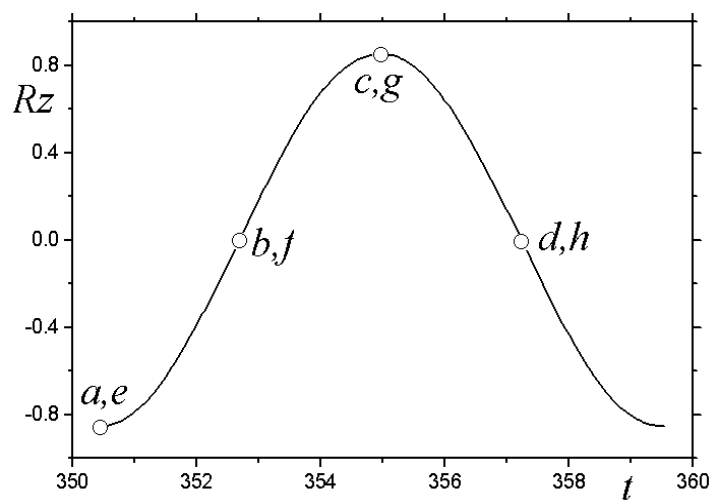

Fig. 2.Parallelepiped transverse load at the vibration period $\mathrm{Rz}(\mathrm{t})$.

It should be noted that up to now there are no fixed requirements for the execution of investigations, especially numerical. So, for example, in building aerodynamics some people still use stationary models. At the same time, it is considered quite acceptable to issue conclusions on building objects based on numerical simulation results. It is doubtless that only a complex approach combining the methods of experimental investigation and numerical simulation is reliable and advisable in the field of architecture and building aerothermodynamics.

Spatial arrangements of model ensembles have been examined in the wind tunnel of the Research Institute of Mechanics of the Moscow State University experimentally and then numerically. A periodic character of flow is demonstrated by the distributions of transverse and longitudinal loads for a rectangular construction. Averaged experimental and predicted local loads at the front, side, and rear surfaces of the construction manifest themselves in considerable rarefaction and pulsation loads on the side edges. The structure of periodic separated flow around the construction is analyzed from the evolution of the pictures of the parameter $\lambda 2=\mathrm{s}^{2}-\mathrm{O}^{2}$. Their notations correspond to the points in the plot of transverse loads during the vibration period (Figs. 2, 3). Within the framework of URANS it is possible to estimate the sizes of vortex structures, as well as to reveal the mechanism of their formation near the body.

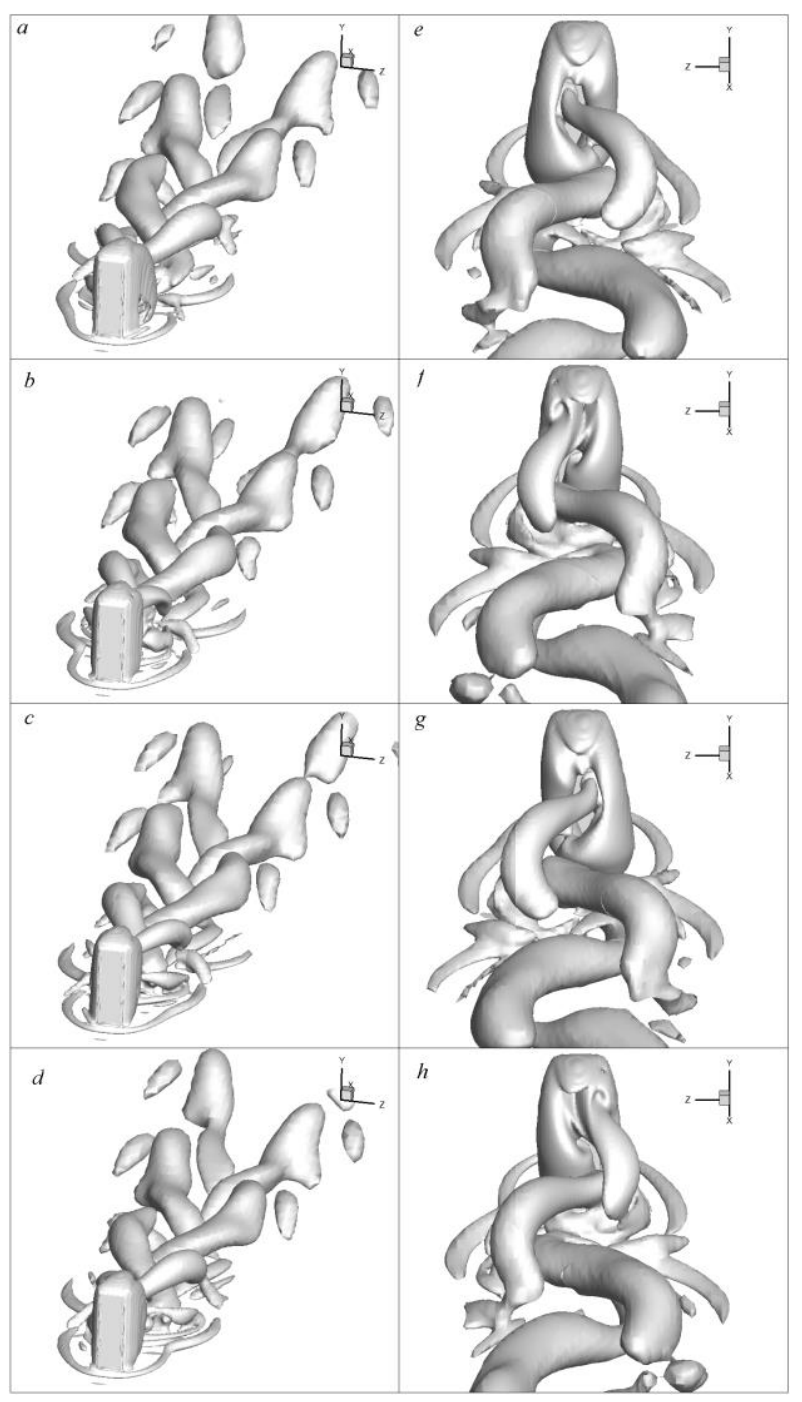

Fig. 3. Visualization of vortex structures in flow around constructions at the moments shown in Fig. 2. a,b,c,d - front view, e,f,g,h - back view.

The results on the flow around a cylinder in a thinwalled housing, which have been obtained at the beginning of the decade, are now refined. It is shown that the optimal location of two windows in front and from behind provides minimum aerodynamic drag within the laminar flow regime.
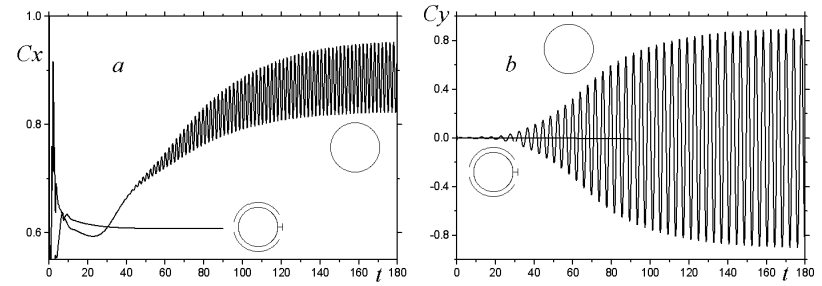

Fig. 4. Comparison of the longitudinal $(a)$ and transverse $(b)$ loads on the circular cylinder with and without windows. $\operatorname{Re}=10^{5}$

An exclusive result - the stabilization of turbulent unsteady wake flow behind the circular cylinder (Fig. 4) has been obtained. The averaged flow patterns at different Reynolds numbers from 45000 to $4.5 \times 10^{6}$ are analyzed. The plot of the experimental behavior of circular cylinder 
drag with increasing Reynolds number also demonstrates the estimated dependence of the cylinder in the housing with windows (Fig. 5). A three-fold drag reduction of the arrangement at high Re numbers is revealed.

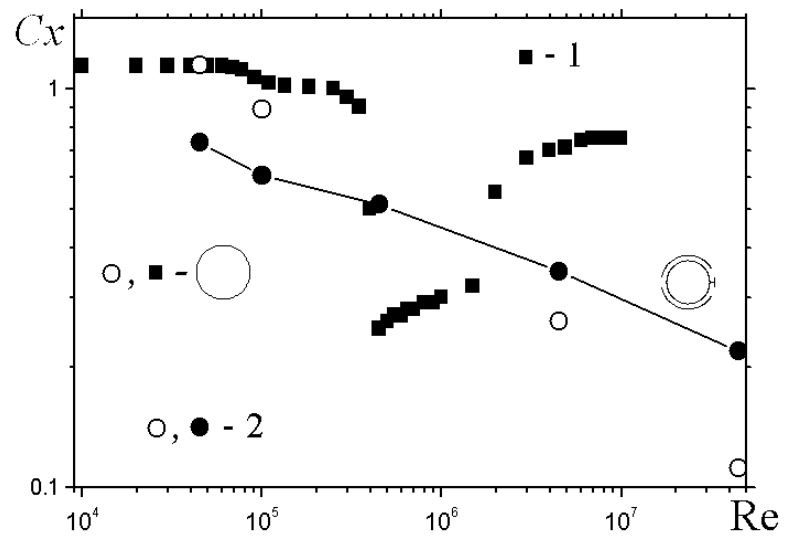

Fig. 5. Comparison of the experimental data (1) and the numerical (2D-URANS+MSST) predictions (2) for the drag coefficients of the circular cylinder and the cylinder in the housing with windows.

The symmetric location of two fans on the backside of the cylinder is considered. The fan model is similar to the one used in Fluent (the fan pressure drop is assigned by the formula $\Delta \mathrm{p}=K s \mathrm{~V}_{\mathrm{n}}^{2}$, where $\mathrm{V}_{\mathrm{n}}$ is the mean-mass velocity). Some destabilization of flow in the body wake is seen. The static pressure distribution pictures show that energy extraction causes the flow to decelerate, but the total drag of the body in this case falls (Fig. 6).
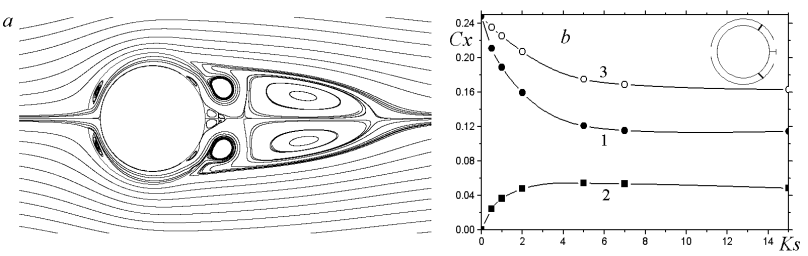

Fig. 6. Flow around the cylinder in the housing with windows at high coefficients $K s(a)$ and averaged drag coefficient vs. $K s$ (b). $1-C x, 2$ - additional drag caused by a fan, 3 - total drag.

\section{Developing MCT and code VP2/3 (velocity - pressure - 2D and 3D) + verification}

Three monographs (Belov et al. 1989; Bystrov et al. 2005; Ermishin, Isaev 2003) have become an information basis for the material presented here. Basic data are the unsteady Reynolds-averaged Navier-Stokes equations (URANS) for an incompressible viscous fluid that was closed by Menter's shear stress transfer model (MSST) in 2003, then it was modified with regard to the streamline curvature influence in the framework of Rodi and Leshtsiner's approach with the additional constant $C_{c}$ equal to 0.02 . The MCT features are mainly outlined in the monograph (Bystrov et al. 2005). A combination of different-scale meshes superimposed on each other enables one not only to discretize the computational domain using the structured meshes of simple topology (close to orthogonal ones) but also to separate differentscale structure elements of flow in the form of boundary and shear layers, von Karman streets, separated flow zones, etc. Thus, computational resources are saved and the high accuracy corresponding to the use of adaptive meshes is provided.

A cube located on a flat wall is also the test known to all when solving building aerodynamics problems. The experiment of J. Castro and his colleagues is concerned with two variants of velocity profiles of the incoming flow with thin and thick boundary layers (Castro, Robins 1977). Here, the first variant is used for comparison, although in computations the boundary layer is considered to be somewhat thicker than in experiment. The self-similar regime is reached at Re varying from $10^{4}$ to $10^{6}$. The transverse load at the cube during the vibration period undergoes sinusoidal changes, its maximum growing from 0.1 to 0.14 with increasing Re. The drag $\mathrm{C}_{x}$ slightly changes with increasing Re. The comparison of the numerical predictions with the experimental data shows that their agreement is good and the use of URANS is also acceptable. Similar comparisons are made for the cylinder (experiment taken from (Devnin 1983)) and for the parallelepiped (experiment made at the Research Institute of Mechanics, Moscow State University) on the plane.

\section{Conclusions}

At present, there is a need to supplement the code VP2/3 with turbulence models and databases.

External and internal conjugated problems on aerothermodynamics of constructions should be solved.

As for ecology, the influence of the relief and green plantations on aerodynamics and ventilation of high-rise buildings should be taken into consideration.

In future, the problems of unsteady aerodynamics and elasticity theory should be solved at a time.

\section{References}

Ermishin, A.; Isaev, S., 2003. Control of flow around vortex cell-containing bodies as applied to the aircrafts of integral arrangement (numerical simulation and experimental investigation). Moscow: MSU Press. $360 \mathrm{p}$.

Babaskin, V.; Isaev, S.; Metov, Kh.; Pyshnyi I.; Chepiga, V., 2002. Wind shear as used in aircrafts (warning system). Saint-Petersburg: Academy of Civil Aviation. 146 p.

Babaskin, V. V.; Isaev, S. A.; Metov, Kh. T.; Chepiga, V. E., 2008. Numerical simulation and the warning system of a hazardous orographical wind shear in airports. All-Russian Scientific and Technical Journal "Flight", 12, pp.27-34.

Baranov, P.; Guvernyuk, S.; Isaev, S.; Kharchenko, V., 2003. Numerical simulation of laminar flow around a cylinder with a co-axial front disc at small and moderate angles of attack using multiblock computational technologies. Aeromechanics and Gasdynamics, 1, pp. 16-27.

Baranov, P.; Isaev, S.; Korolev, E.; Usachov, A., 2005. Ventilation computation of open embrasures of road tunnels using the numerical simulation methods. Metro and Tunnels, 6, pp. 13-18.

Belov, I.; Isaev, S.; Korобкоv, V., 1989. Tasks and calculation methods of separated incompressible liquid flows. Leningrad: Sudostroyeniye. $256 \mathrm{p}$. 
Bungov, V.; Isaev, S.; Kharchenko, V., 1998. Calculation of the wind action on a cooling tower in the presence of devices for equalizing the ground flow. Journal of Engineering Physics and Thermophysics, 71(5), pp. 852-857. http://dx.doi.org/10.1007/BF02681636

Bystrov, Yu.; Isaev, S.; Kudryavtsev, N.; Leontiev, A., 2005. Numerical simulation of vortex heat transfer enhancement in tube bundles, Saint-Petersburg: Sudostrouyeniye. 398 p.

Castro, I.; Robins, A., 1977. The flow around a surfacemounted cube in uniform and turbulent streams. Journal of Fluid Mechanics, 79(2), pp. 307-335. http://dx.doi.org/10.1017/S0022112077000172

Devnin, S., 1983. Aerohydromechanics of high-drag constructions. Handbook. Leningrad: Sudostroyeniye. 320 p.

Isaev, S.; Zhdanov, V.; Niemann, H.-J., 2002. Numerical study of the bleeding effect on the aerodynamic characteristics of a circular cylinder. Journal of Wind Engineering and Industrial Aerodynamics, 90(11), pp. 1217-1226. http://dx.doi.org/10.1016/S0167-6105(02)00253-2

Isaev, S.; Baranov, P.; Usachov, A.; Mitrofovich, V.; Kolosov, A.; Ponomarev, M., 2003. Numerical modeling of turbulent flow inside a wind-driven plant with allowance for the forces on the impeller. Journal of Engineering Physics and Thermophysics, 76(6), pp. 1246-1250. http://dx.doi.org/10.1023/B:JOEP.0000012025.23698.1d

Isaev, S.; Sudakov, A.; Baranov, P.; Usachov, A.; Strizhak, S.; Lokhansky, Ya.; Guvernyuk, S., 2009. Development, verification, and application of the multiblock computational technology-based parallel open code VP2/3 for solving fundamental, applied, and maintenance problems of aeromechanics and thermal physics. Bulletin of South Ural State University. Series "Mathematical Modeling and Programming", 17(3), pp. 59-72.

Isaev, S.; Kornev, N.; Leontiev, A.; Hassel, E., 2010(a). Influence of the Reynolds number and the spherical dimple depth on the turbulent heat transfer and hydraulic loss in a narrow channel. International Journal of Heat and Mass Transfer, 53(1-3), pp. 178-197. http://dx.doi.org/10.1016/j.ijheatmasstransfer.2009.09.042
Isaev, S.; Leontiev, A., 2010(b). Problems of simulating tornado-like heat transfer turbulent flow past a dimpled relief on a narrow channel wall. Journal of Engineering Physics and Thermophysics, 83(4), pp. 783-793. http://dx.doi.org/10.1007/s10891-010-0404-5

Isaev, S.; Vatin N.; Baranov, P.; Sudakov, A.; Usachov, A.; Egorov, V., 2013(a). Development and verification of multiblock computational technologies for solving unsteady problems of building aerodynamics of high-rise buildings within the URANS approach. Journal of Engineering Physics and Thermophysics, pp. 86(1), pp. 103-109

Isaev, S.; Vatin, N.; Lebiga, V.; Zinoviev, V.; Chang Keh-Chin; Miau, Jiun-Jih., 2013(b). Problems and methods of numerical and experimental investigations of aerodynamics for high-rise constructions in the coastal region "sea-land". Journal of Civil Engineering, 2, pp. 54-61.

Kalinin, E.; Dreitser, G.; Kopp, I.; Myakochin, A., 1998. Effective heat transfer surfaces. Moscow: Energoatomizdat. 408 p.

Lawson, T., 2001. Building aerodynamics. London: Imperial College Press. 286 p.

Menter, F., 1993. Zonal two equation k- $\omega$ turbulence models for aerodynamic flows. AIAA, Paper No 93-2906:21.

Menter, F.; Kuntz, M.; Langtry, R., 2003. Ten years of industrial experience with the SST turbulence model. Turbulence, Heat and Mass Transfer 4. Ed. K.Hajalic, Y.Nogano, M.Tummers. Begell House, Inc.

Usachov, A.; Kolesnikov, A.; Ismail, Siyavoshi, M.; Isaev, S., 2004. Ventilation system and numerical simulation of the propagation of smoke and thermal energy at fire in the Alborz mountain tunnel in Iran. Underground Areas of the World, 1, pp. 49-52.

\section{Acknowledgements}

The work was financially supported by RFBS (Projects 11-01-00039, 12-08-90001 and 13-08-90468) and BFFR (Project T12R-217). 